\title{
A coherent approach to non-integer order derivatives
}

\author{
Manuel Duarte Ortigueira*,1 \\ UNINOVA, Campus da FCT da UNL, Quinta da Torre, 2825-114 Monte da Caparica, Portugal
}

Received 5 May 2005; received in revised form 7 December 2005

Available online 2 March 2006

\begin{abstract}
The relation showing that the Grünwald-Letnikov and generalised Cauchy derivatives are equal is presented. This establishes a bridge between two different formulations and simultaneously between the classic integer order derivatives and the fractional ones. Starting from the generalised Cauchy derivative formula, new relations are obtained, namely a regularised version that makes the concept of pseudo-function appear naturally without the need for a rejection of any infinite part. From the regularised derivative, new formulations are deduced and specialised first for the real functions and afterwards for functions with Laplace transforms obtaining the definitions proposed by Liouville. With these tools suitable definitions of fractional linear systems are obtained.
\end{abstract}

(C) 2006 Elsevier B.V. All rights reserved.

Keywords: Fractional difference; Fractional derivative; Fractional linear system; Differintegrator

\section{Introduction}

Fractional calculus, while over 300 years old, is still a young field, rich in opportunities for fundamental discoveries. In recent years fractional calculus has been rediscovered by scientists and engineers and applied in an increasing number of fields, namely in the areas of electromagnetism, control engineering, and signal processing. The increase in the number of physical and engineering processes that are best described by fractional differential equations has motivated out its study. This led to an enrichment of fractional calculus with

\footnotetext{
*Tel.: + 35112948520 ; fax: + 35112957786 .

E-mail addresses: mdortigueira@uninova.pt, mdo@dee.fct.unl.pt.

${ }^{1}$ Also with INESC_ID, R. Alves Redol, 9, 2 , Lisbon, Portugal.
}

new approaches that however brought contributions to a somehow chaotic state of the art. In fact, there are several definitions that lead to different results, making difficult the establishment of a systematic theory of fractional linear systems in agreement with the current practice. RiemannLiouville, Caputo, Grünwald-Letnikov, Hadamard, Marchaud, are some of the known definitions $[1-9,16,17]$. Although from a purely mathematical point of view it is legitimate to accept and even use one or all, from the point of view of applications the situation is different. In our case of signal processing applications, we should accept only the definitions that might lead to a fractional systems theory coherent with the usual practice, and accepted notions and concepts such as the impulse response and transfer function. In previous papers [7-9] we made some contributions toward this goal; 
however, despite these developments, several topics remained without a clear and concise formulation, namely and surprisingly, the definition of fractional derivative $^{2}$ suitable for our interests. In [9] we addressed this problem and proposed a solution based on a reasoning that led to the use of the Grünwald-Letnikov and the so-called generalised functions (Cauchy), forward and backward derivatives. $^{3}$ These choices were motivated by four main reasons: they do not need superfluous derivative computations, do not insert unwanted initial conditions, are more flexible and allow sequential computations. However, in this earlier approach we did not present a coherent mathematical reasoning or a connection between the two formulations. In this paper, we propose to provide these missing links. In facing this problem, we assume as a starting point the definitions of direct and reverse fractional differences and their integral representations [11-13]. From these representations and using the asymptotic properties of the Gamma function, we obtained in $[12,13]$ a generalised Cauchy integral as a unified formulation for any order derivative. As we proved in $[12,13]$ :

The generalised Cauchy derivative is equal to the Grünwald-Letnikov fractional derivative.

When trying to compute the Cauchy integral using the Hankel contour we conclude that:

- the integral has two terms: one corresponds to a derivative and the other to a primitive,

- the exact computation leads to a regularised integral, generalising the well-known concept of pseudo-function, but without rejecting any infinite part,

- the definition implies causality.

The forward and backward derivatives emerge here as very special cases. We will study them for the case of functions with Laplace transforms. This leads us to define a causal and an anti-causal fractional linear differintegrators both with transfer function equal to $s^{\alpha}, \alpha \in R$, and to compute their corresponding impulse responses.

\footnotetext{
${ }^{2}$ Here we will speak mainly in terms of the fractional or noninteger order derivative, avoiding the use of fractional integral, because the calculus of the derivative may be done through an integral. So the word integral would appear with two different assertions. We will use derivative of negative order or primitive.

${ }^{3}$ As pointed out by Dugowson [10] these were already proposed by Liouville.
}

The paper outline is as follows. In Section 2, we present the way we followed from the fractional differences to the fractional derivative defined in the complex plane, obtaining the well-known Cauchy integral. The analysis of the generalised Cauchy integral is done in Section 3 with the help of the Hankel contour. We present a general formula and two special cases valid for real variable functions. We exemplify with the exponential function and present the forward and backward derivatives as special cases valid for real functions in Section 4. In Section 5, we treat the case of functions with Laplace transform and obtain the formulae we named before by Cauchy differintegrations [9], but that must be named as Liouville differintegrations, since they were proposed first by Liouville [10] These are suitable for fractional signals and systems studies [7] since they allow a generalisation of known concepts without meaningful changes as shown in Section 6. In Section 7 we will present some conclusions. At last in Appendix we obtain the impulse response for the differintegrator defined as a linear system with transfer function equal to $s^{\alpha}, \alpha \in R$.

Caution: In this paper we deal with a multivalued expression $z^{\alpha}$. As is well known, to define a function we have to fix a branch cut line and choose a branch (Riemann surface). It is a common procedure to choose the negative real half-axis as branch cut line. In what follows we will assume that we adopt the principal branch and assume that the obtained function is continuous above the branch cut line. With this, we will write $(-1)^{\alpha}=\mathrm{e}^{j \alpha \pi}$.

\section{From differences to derivatives}

\subsection{Difference definitions}

Let $f(z)$ be a complex variable function and introduce $\Delta_{\mathrm{d}}$ and $\Delta_{\mathrm{r}}$ as finite "direct" and "reverse" $[12,13]$ order one differences defined by

$$
\Delta_{\mathrm{d}} f(z)=f(z)-f(z-h)
$$

and

$$
\Delta_{\mathrm{r}} f(z)=f(z+h)-f(z)
$$

with $h \in C$ and we assume that $\operatorname{Re}(h)>0$. For any order (including the negative integer case) we have $[9,11]$ :

$$
\Delta_{\mathrm{d}}^{\alpha} f(z)=\sum_{k=0}^{\infty}(-1)^{k}\left(\begin{array}{l}
\alpha \\
k
\end{array}\right) f(z-k h)
$$


and

$\Delta_{\mathrm{r}}^{\alpha} f(z)=(-1)^{\alpha} \sum_{k=0}^{\infty}(-1)^{k}\left(\begin{array}{l}\alpha \\ k\end{array}\right) f(z+k h)$,

where $\left(\begin{array}{l}\alpha \\ k\end{array}\right)$ are the binomial coefficients.

\subsection{On the Grünwald-Letnikov differintegrations}

Divide (3) by $h^{\alpha}$ to obtain the fractional incremental ratio. Performing the computation of its limit as $h \rightarrow 0^{+},{ }^{4}$ we obtain the direct GrünwaldLetnikov derivative given by

$D_{\mathrm{d}}^{\alpha} f(z)=\lim _{h \rightarrow 0+} \frac{\sum_{k=0}^{\infty}(-1)^{k}\left(\begin{array}{l}\alpha \\ k\end{array}\right) f(z-k h)}{h^{\alpha}}$,

where $h$ is any complex number in the right-hand complex plane. Making a substitution $h \rightarrow-h$ \{or using (4)\}, we obtain the reverse GrünwaldLetnikov derivative:

$D_{\mathrm{r}}^{\alpha} f(z)=\lim _{h \rightarrow 0+}(-1)^{\alpha} \frac{\sum_{k=0}^{\infty}(-1)^{k}\left(\begin{array}{l}\alpha \\ k\end{array}\right) f(z+k h)}{h^{\alpha}}$.

Expression (5) corresponds to the so-called lefthand sided Grünwald-Letnikov fractional derivative $[15,16]$ while $(6)$ has the extra factor $(-1)^{\alpha}$, when compared with the right-hand sided Grünwald-Letnikov fractional derivative [10]. We maintain that this factor must be retained and continue to call the pairs defined by (5) and (6) as GrünwaldLetnikov fractional derivatives. It is interesting to remark that these definitions were proposed first by Liouville [10].

Although we are not concerned here with existence problems, we must refer that in general we can have the direct derivative without existence of the reverse one and vice versa. For example, let us apply both definitions to the function $f(z)=\mathrm{e}^{a z}$. If $\operatorname{Re}(a)>0$, expression (5) converges to $D_{\mathrm{d}}^{\alpha} f(z)=$ $a^{\alpha} \mathrm{e}^{a z}$, while (6) diverges. On the other hand, if $f(z)=\mathrm{e}^{-a z}$ Eq. (5) diverges while (6) converges to $D_{\mathrm{r}}^{\alpha} f(z)=(-a)^{\alpha} \mathrm{e}^{-a z}$. This suggests that (5) and (6) should be adopted for right and left functions, ${ }^{5}$ respectively, in agreement with Liouville reasoning [10]. In particular, they should be used for the

\footnotetext{
${ }^{4} 0^{+}$means that $\operatorname{Re}(h)>0$.

${ }^{5} \mathrm{We}$ say that $f(t)$ is a right [left] function if $f(-\infty)=0$ $[f(+\infty)=0]$.
}

functions such that $f(z)=0$ for $\operatorname{Re}(z)<0$ and $f(z)=$ 0 for $\operatorname{Re}(z)>0$, respectively. It is interesting to remark that, if $z$ and $h$ are real, in (5) we are using the current and past values of the function: it is a causal derivative; on the other hand, in (6) we use the current and future values: it is an anti-causal derivative.

\subsection{Integral representations for the differences}

Assume that $f(z)$ is analytic inside and on an infinite integration path that encircles the points $t=$ $z-k h$ in the direct case and $t=z+k h$ in the corresponding reverse case, with $k \in Z_{0}^{+}$. The results stated in (3) and (4) can be interpreted in terms of the residue theorem and written as [11-13]

$$
\Delta_{\mathrm{d}}^{\alpha} f(z)=\frac{\Gamma(\alpha+1)}{2 \pi j h} \int_{C_{\mathrm{d}}} f(w) \frac{\Gamma((w-z) / h)}{\Gamma([(w-z) / h]+\alpha+1)} \mathrm{d} w
$$

and

$$
\begin{aligned}
\Delta_{\mathrm{r}}^{\alpha} f(z)= & \frac{(-1)^{\alpha+1} \Gamma(\alpha+1)}{2 \pi j h} \\
& \times \int_{C_{\mathrm{r}}} f(w) \frac{\Gamma((z-w) / h)}{\Gamma([(z-w) / h]+\alpha+1)} \mathrm{d} w .
\end{aligned}
$$

Remark that:

(a) One turns into the other with the substitution $h \rightarrow-h$, as expected.

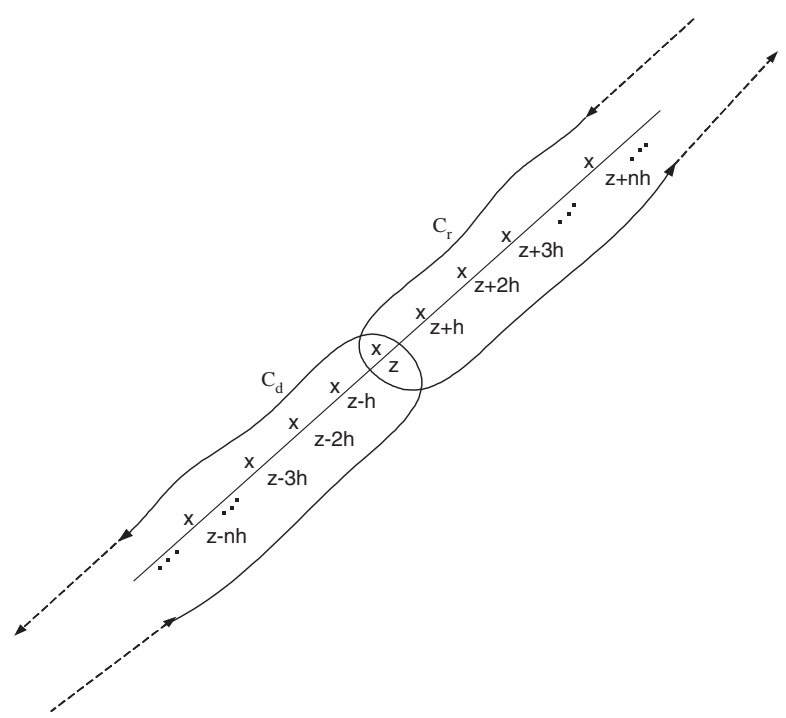

Fig. 1. Integration paths and poles for the integral representation of fractional order differences. 
(b) The integration path must encircle the halfstraight line that contains all the poles. This can be done with a $U$ shaped contour like those shown in Fig. 1.

(c) The direct and reverse differences are not equal.

\subsection{Generalising the Cauchy formula}

The ratio of two gamma functions $(\Gamma(s+$ $a)) /(\Gamma(s+b))$ has the expansion $[14,15]$ :

$\frac{\Gamma(s+a)}{\Gamma(s+b)}=s^{a-b}\left[1+\sum_{1}^{N} c_{k} s^{-k}+O\left(s^{-N-1}\right)\right]$

as $|s| \rightarrow \infty$, uniformly in every sector that excludes the negative real half-axis. As shown in [12] we can use the above expansion to obtain for the fractional incremental ratios the following representations:

$\frac{\Delta_{\mathrm{d}}^{\alpha} f(z)}{h^{\alpha}}=\frac{\Gamma(\alpha+1)}{2 \pi j} \int_{C_{\mathrm{d}}} f(w) \frac{1}{(w-z)^{\alpha+1}} \mathrm{~d} w+h \cdot g_{1}(h)$

and

$\frac{\Delta_{\mathrm{r}}^{\alpha} f(z)}{h^{\alpha}}=\frac{\Gamma(\alpha+1)}{2 \pi j} \int_{C_{\mathrm{r}}} f(w) \frac{1}{(w-z)^{\alpha+1}} \mathrm{~d} w+h \cdot g_{2}(h)$,

where $g_{1}(h)$ and $g_{2}(h)$ remain finite when $h$ decreases. Allowing $h \rightarrow 0^{+}$, we obtain the direct and reverse generalised Cauchy integrals:

$D_{\mathrm{d}}^{\alpha} f(z)=\frac{\Gamma(\alpha+1)}{2 \pi j} \int_{C_{\mathrm{d}}} f(w) \frac{1}{(w-z)^{\alpha+1}} \mathrm{~d} w$

and

$D_{\mathrm{r}}^{\alpha} f(z)=\frac{\Gamma(\alpha+1)}{2 \pi j} \int_{C_{\mathrm{r}}} f(w) \frac{1}{(w-z)^{\alpha+1}} \mathrm{~d} w$,

that represent the $\alpha$ order derivative. When $\alpha=N$ (a positive integer), both the derivatives are equal and coincide with the usual Cauchy derivative. In the fractional case we have different solutions, since we are using a different integration path. We can then define a generalised Cauchy integral by

$D^{\alpha} f(z)=\frac{\Gamma(\alpha+1)}{2 \pi j} \int_{C} f(w) \frac{1}{(w-z)^{\alpha+1}} \mathrm{~d} w$,

where $C$ is any $\mathrm{U}$ shaped contour that encircles the half-straight line starting at $z$ that is the branch cut line of $w^{-\alpha-1}$. This formula has been used in fractional calculus $[4,15,16]$ for defining what Campos calls the Liouville system [1]. Some authors use a different version for branched functions that uses a closed integration path: it is the called Riemann system $[1,2,15]$. According to the theory we just developed this way of introducing derivatives does not conform to the assumptions behind (14). So, it does not lead to valid derivatives: it results in what we call pseudo-derivatives. In applying (14) to a branched function, the integration path must be inside the analyticity domain of the function. This means that the branch cut line used in (14) cannot cut any other branch cut lines used to define the function.

\section{Analysis of Cauchy formula}

Consider the generalised Cauchy formula (14) and rewrite it in a more convenient format obtained by a simple translation:

$D^{\alpha} f(z)=\frac{\Gamma(\alpha+1)}{2 \pi j} \int_{C} f(w+z) \frac{1}{w^{\alpha+1}} \mathrm{~d} w$,

where we assume that $f(z)$ is analytic in a region that contains the contour $C$. Here we will choose $C$ as a special integration path: the Hankel contour represented in Fig. 2. We assume that it surrounds the selected branch cut line. This is described by $x . \mathrm{e}^{j \theta}$, with $x \in R^{+}$and $\theta \in[0,2 \pi)$. The circle has a radius equal to $\rho$ small enough to allow it to stay inside the region of analyticity of $f(z)$.

With this contour, we can decompose (15) into three integrals along the two half-straight lines and the circle. It is interesting to remark that if $\alpha$ is a positive integer, the integrals along the straight lines cancel out and it remains the integral over the

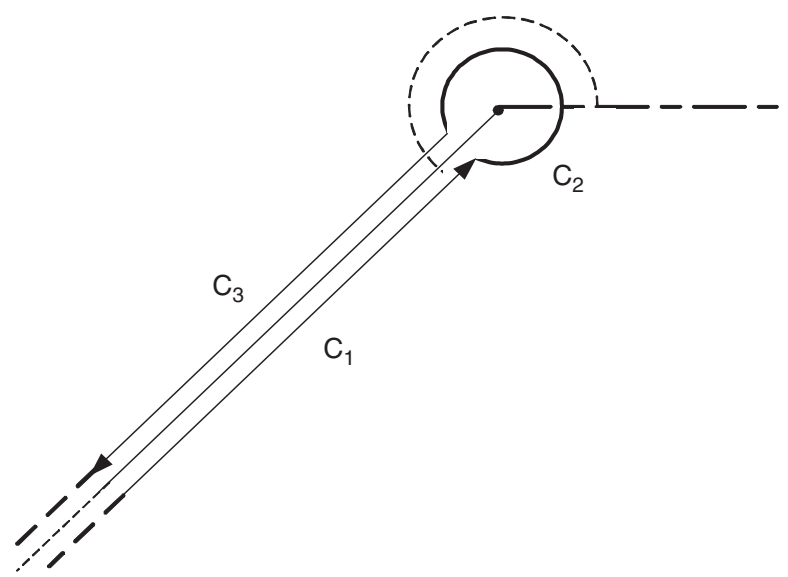

Fig. 2. The Hankel contour used in computing the derivative defined in Eq. (15). 
circle: we obtain the usual Cauchy formula. If $\alpha$ is a negative integer, the integral along the circle is zero and we are led to the well-known repeated integration formula $[3,4,15,16]$. In the general $\alpha$ case we need the two terms. Let us decompose the above integral using the Hankel contour. For reducing steps, we will assume already that the straight lines are infinitely near to each other. We have, then:

$$
\begin{aligned}
D^{\alpha} f(z)= & \frac{\Gamma(\alpha+1)}{2 \pi j}\left[\int_{C 1}+\int_{C 2}+\int_{C 3}\right] \\
& \times f(w+z) \frac{1}{w^{\alpha+1}} \mathrm{~d} w .
\end{aligned}
$$

Over $C_{1}$ we have $w=x \cdot \mathrm{e}^{j(\theta-2 \pi)}$, while over $C_{3}$ we have $w=x . \mathrm{e}^{\mathrm{j} \theta}$, with $x \in R^{+}$; over $C_{2}$ we have $w=\rho \mathrm{e}^{j \varphi}$, with $\varphi \in(\theta-2 \pi, \theta)$. We can write, at last:

$$
\begin{aligned}
D^{\alpha} f(z)= & \frac{\Gamma(\alpha+1)}{2 \pi j}\left[\int_{\infty}^{\rho} f\left(x \cdot \mathrm{e}^{j(\theta-2 \pi)}+z\right) \frac{\mathrm{e}^{-j \alpha(\theta-2 \pi)}}{x^{\alpha+1}} \mathrm{~d} x\right. \\
& \left.+\int_{\rho}^{\infty} f\left(x \cdot \mathrm{e}^{j \theta}+z\right) \frac{\mathrm{e}^{-j \alpha \theta}}{x^{\alpha+1}} \mathrm{~d} x\right] \\
& +\frac{\Gamma(\alpha+1)}{2 \pi j} \frac{1}{\rho^{\alpha}} \int_{\theta-2 \pi}^{\theta} f\left(\rho \cdot \mathrm{e}^{j \varphi}+z\right) \\
& \times \mathrm{e}^{-j \alpha \varphi} j \mathrm{~d} \varphi .
\end{aligned}
$$

For the first term, we have:

$$
\begin{aligned}
\int_{\infty}^{\rho} & f\left(x \cdot \mathrm{e}^{j(\theta-2 \pi)}+z\right) \frac{\mathrm{e}^{-j \alpha(\theta-2 \pi)}}{x^{\alpha+1}} \mathrm{~d} x \\
+ & \int_{\rho}^{\infty} f\left(x \cdot \mathrm{e}^{j \theta}+z\right) \frac{\mathrm{e}^{-j \alpha \theta}}{x^{\alpha+1}} \mathrm{~d} x \\
= & {\left[-\mathrm{e}^{-j \alpha(\theta-2 \pi)}+\mathrm{e}^{-j \alpha \theta}\right] \int_{\rho}^{\infty} f\left(x \cdot \mathrm{e}^{j \theta}+z\right) \frac{1}{x^{\alpha+1}} \mathrm{~d} x } \\
= & \mathrm{e}^{-j \alpha \theta}\left[1-\mathrm{e}^{j 2 \pi \alpha}\right] \int_{\rho}^{\infty} f\left(x \cdot \mathrm{e}^{j \theta}+z\right) \frac{1}{x^{\alpha+1}} \mathrm{~d} x \\
= & -\mathrm{e}^{j \alpha(\pi-\theta)} \cdot 2 j \sin (\alpha \pi) \\
& \times \int_{\rho}^{\infty} f\left(x \cdot \mathrm{e}^{j \theta}+z\right) \frac{1}{x^{\alpha+1}} \mathrm{~d} x
\end{aligned}
$$

where we assumed that $f\left(x \cdot \mathrm{e}^{j(\theta-2 \pi)}+z\right)=$ $f\left(x . \mathrm{e}^{j \theta}+z\right)$, because $f(z)$ is analytic.

For the second term, we begin by noting that the analyticity of the function $f(z)$ allows us to write:

$f\left(x . \mathrm{e}^{j \theta}+z\right)=\sum_{0}^{\infty} \frac{f^{(n)}(z)}{n !} x^{n} \mathrm{e}^{j n \theta}$ for $x<r \in R^{+}$. We have, then:

$$
\begin{aligned}
j \frac{1}{\rho^{\alpha}} & \int_{\theta-2 \pi}^{\theta} f\left(\rho \cdot \mathrm{e}^{j \varphi}+z\right) \mathrm{e}^{-j \alpha \varphi} \mathrm{d} \varphi \\
& =j \frac{1}{\rho^{\alpha}} \sum_{0}^{\infty} \frac{f^{(n)}(z)}{n !} \rho^{n} \int_{\theta-2 \pi}^{\theta} \mathrm{e}^{j(n-\alpha) \varphi} \mathrm{d} \varphi .
\end{aligned}
$$

Performing the integration, we have:

$$
\begin{aligned}
j & \frac{1}{\rho^{\alpha}} \int_{\theta-2 \pi}^{\theta} f\left(\rho \cdot \mathrm{e}^{j \varphi}+z\right) \mathrm{e}^{-j \alpha \varphi} \mathrm{d} \varphi \\
& =-j \cdot \mathrm{e}^{j \alpha \pi} \sum_{0}^{\infty} \frac{f^{(n)}(z)}{n !} \rho^{n-\alpha} \mathrm{e}^{j(n-\alpha) \theta} \frac{2 \cdot \sin [(n-\alpha) \pi]}{(n-\alpha)} \\
& =-2 j \cdot \mathrm{e}^{j \alpha \pi} \sin (\alpha \pi) \sum_{0}^{\infty} \frac{f^{(n)}(z)}{n !} \frac{\mathrm{e}^{j(n-\alpha) \theta} \rho^{n-\alpha}}{(n-\alpha)}
\end{aligned}
$$

But the summation in the last expression can be written in another interesting format:

$$
\begin{aligned}
\sum_{0}^{\infty} & \frac{f^{(n)}(z)}{n !} \cdot \frac{\mathrm{e}^{j(n-\alpha) \theta} \rho^{n-\alpha}}{(n-\alpha)} \\
= & -\sum_{0}^{N} \frac{f^{(n)}(z)}{n !} \mathrm{e}^{j n \theta} \int_{\rho}^{\infty} x^{n-\alpha-1} \mathrm{~d} x \\
& +\sum_{N+1}^{\infty} \frac{f^{(n)}(z)}{n !} \frac{\mathrm{e}^{j n \theta} \rho^{n-\alpha}}{(n-\alpha)}
\end{aligned}
$$

where $N=\lfloor\alpha\rfloor{ }^{6}$ Substituting it in (21) and joining to (18) we can write:

$$
\begin{aligned}
D^{\alpha} f(z)= & K \cdot \int_{\rho}^{\infty} \frac{\left[f\left(x \cdot \mathrm{e}^{j \theta}+z\right)-\sum_{0}^{N} \frac{f^{(n)}(z)}{n !} \mathrm{e}^{j n \theta} x^{n}\right]}{x^{\alpha+1}} \mathrm{~d} x \\
& -K \cdot \sum_{N+1}^{\infty} \frac{f^{(n)}(z)}{n !}(-1)^{n} \frac{\rho^{n-\alpha}}{(n-\alpha)} .
\end{aligned}
$$

If $\alpha<0$, we make the inner summation equal to zero. Using the reflection formula of the gamma function we obtain for $K$ :

$$
K=-\frac{\Gamma(\alpha+1) \mathrm{e}^{j(\pi-\theta) \alpha}}{\pi} \sin (\alpha \pi)=\frac{\mathrm{e}^{j(\pi-\theta) \alpha}}{\Gamma(-\alpha)} .
$$

\footnotetext{
${ }^{6}\lfloor\alpha\rfloor$ means "the greatest integer less than or equal to $\alpha$ ".
} 
Now let $\rho$ go to zero. The second term on the right-hand side in (22) goes to zero and we obtain:

$$
\begin{aligned}
D^{\alpha} f(z)= & \frac{\mathrm{e}^{j(\pi-\theta) \alpha}}{\Gamma(-\alpha)} \\
& \times \int_{0}^{\infty} \frac{\left[f\left(x \cdot \mathrm{e}^{j \theta}+z\right)-\sum_{0}^{N} \frac{f^{(n)}(z)}{n !} \mathrm{e}^{j n \theta} x^{n}\right]}{x^{\alpha+1}} \mathrm{~d} x,
\end{aligned}
$$

that is valid for any $\alpha \in R$. It is interesting to remark that (24) is nothing else but a generalisation of the "pseudo-function" notion [17], but valid for an analytic function in a non-compact region of the complex plane. Relation (24) represents a regularised fractional derivative that has some similarities with the Marchaud derivative [15]: for $0<\alpha<1$, they are equal. A special case of (24), for $\theta=\pi$, can be found in [15]. It was obtained using the concept of finite part Hadamard integral. However, (24) appears naturally from the generalised Cauchy derivative without having to reject any infinite part.

If one puts $w=x \cdot \mathrm{e}^{\mathrm{j} \theta}$, we can write:

$$
\begin{aligned}
D^{\alpha} f(z)= & \frac{1}{\Gamma(-\alpha)} \mathrm{e}^{j \pi \alpha} \\
& \times \int_{\gamma} \frac{\left[f(w+z)-\sum_{0}^{N} \frac{f^{(n)}(z)}{n !} w^{n}\right]}{w^{\alpha+1}} \mathrm{~d} w,
\end{aligned}
$$

where $\gamma$ is a half-straight line starting at $w=0$. As we can conclude there are infinitely many ways of computing the derivative of a given function: these are defined by the chosen branch cut lines. However, this does not mean that we have infinitely many different derivatives. It is not hard to see that all the branch cut lines belonging to a given region of analyticity of the function are equivalent and lead to the same result unless the integral may be divergent if the function increases without bound. The results just obtained assume that $f(z)$ is analytic in the region inside and on the integration path. If we are dealing with functions with poles or branch points, these must be excluded from that region.

\section{Examples}

\subsection{The exponential function}

To illustrate the previous assertions we are going to consider the case of the exponential function. Let $f(z)=\mathrm{e}^{a z}$, with $a \in R^{+}$. Inserting it into (24), it becomes

$$
\begin{aligned}
D^{\alpha} f(z)= & \frac{1}{\Gamma(-\alpha)} \mathrm{e}^{j(\pi-\theta) \alpha} \mathrm{e}^{a z} \\
& \times \int_{0}^{\infty} \frac{\left[\mathrm{e}^{a x \cdot \mathrm{e}^{j \theta}}-\sum_{0}^{N} \frac{a^{n}}{n !} \mathrm{e}^{j n \theta} x^{n}\right]}{x^{\alpha+1}} \mathrm{~d} x .
\end{aligned}
$$

With a variable change $\tau=-a x \mathrm{e}^{j \theta}$, the above equation gives:

$D^{\alpha} f(z)=\frac{1}{\Gamma(-\alpha)} a^{\alpha} \mathrm{e}^{a z} \int_{0}^{-\infty \cdot a \mathrm{e}^{j \theta}} \frac{\left[\mathrm{e}^{-\tau}-\sum_{0}^{N} \frac{(-1)^{n}}{n !} \tau^{n}\right]}{\tau^{\alpha+1}} \mathrm{~d} \tau$,

where the integration path is half-straight line that forms an angle equal to $\theta$ with the positive real axis. The integral in (27) is almost the generalised Gamma function definition presented in [18] and is a generalisation of Euler integral representation for the gamma function. But this requires integration along the positive real axis. However, as shown in [14] the integration can be done along a ray with an angle in the interval ]0, $\pi / 2[$. This implies that we must have $\operatorname{Re}\left(-a \mathrm{e}^{\mathrm{j} \theta}\right)>0$. We have then:

$\begin{cases}a>0 & \cos (\theta)<0, \\ a<0 & \cos (\theta)>0 .\end{cases}$

This means that if $a>0$, the branch cut line must belong to left-hand half-plane; while if $a>0$, the branch cut line must belong to right-hand halfplane. As the integrand is analytic outside the branch cut line, we can integrate along the positive real axis. So, we can write:

$D^{\alpha} f(z)=\frac{1}{\Gamma(-\alpha)} a^{\alpha} \mathrm{e}^{a z} \int_{0}^{\infty} \frac{\left[\mathrm{e}^{-\tau}-\sum_{0}^{N} \frac{(-1)^{n}}{n !} \tau^{n}\right]}{\tau^{\alpha+1}} \mathrm{~d} \tau$.

The integral defines the value of the gamma function $\Gamma(-\alpha)$ [18] if we maintain the convention made before: when $\alpha<0$ the summation is zero. We then obtain:

$D^{\alpha} \mathrm{e}^{a z}=a^{\alpha} \mathrm{e}^{a z}$

as expected. In a particular limiting case, $a \rightarrow 0$, we obtain $D^{\alpha} 1=0$ if $\alpha>0$. If $\alpha<0$, it is infinite. The Grünwald-Letnikov definitions allow us to obtain 
the same conclusions as seen. We must be careful in using (29). In fact, in a first glance, we could be led to use it to compute the derivatives of functions like $\sin (z), \cos (z), \sinh (z)$ and $\cosh (z)$. But if we have in mind our reasoning we can conclude immediately that functions do not have finite derivatives if $z \in C$.

In fact they use simultaneously the exponentials $\mathrm{e}^{z}$ and $\mathrm{e}^{-z}$ which derivatives cannot exist simultaneously, as we just saw. However, we can conclude that functions expressed by Dirichlet series $f(t)=\sum_{0}^{\infty} a_{k} \mathrm{e}^{\lambda_{k} t}$ with all the $\operatorname{Re}\left(\lambda_{k}\right)$ positive or all negative have finite derivatives given by $f^{(\alpha)}(t)=$ $\sum_{0}^{\infty} a_{k}\left(\lambda_{k}\right)^{\alpha} \mathrm{e}^{\lambda_{k} t}$. In particular functions with Laplace transform with region of convergence in the right or left half-planes have fractional derivatives. We will return to this case in a later section.

Another interesting case is the cisoid $f(t)=\mathrm{e}^{j \omega t}$, $\omega \in R^{+}$. Inserting it into (24) again, it becomes

$$
\begin{aligned}
D^{\alpha} f(t)= & \frac{1}{\Gamma(-\alpha)} \mathrm{e}^{j(\pi-\theta) \alpha} \mathrm{e}^{j \omega t} \\
& \times \int_{0}^{\infty} \frac{\left[\mathrm{e}^{j \omega x \cdot \mathrm{e}^{j \theta}}-\sum_{0}^{N} \frac{(j \omega)^{n}}{n !} \mathrm{e}^{j n \theta} x^{n}\right]}{x^{\alpha+1}} \mathrm{~d} x .
\end{aligned}
$$

With $\theta=\pi / 2, j \omega \mathrm{e}^{j \theta}=-\omega$ and we obtain easily

$D^{\alpha} f(t)=(j \omega)^{\alpha} \mathrm{e}^{j \omega t}$.

It is not difficult to see that (31) remains valid if $\omega<0$. We only have to put $\theta=-\pi / 2$. We can conclude then that:

$D^{\alpha} \cos (\omega t)=\omega^{\alpha} \cos (\omega t+\alpha \pi / 2)$.

For $\sin (\omega t)$, it is similar.

\subsection{The power function}

Let $f(z)=z^{\beta}$, with $\beta \in R$. If $\beta>\alpha$, we conclude immediately that $D^{\alpha}\left[z^{\beta}\right]$ defined for every $z \in C$ does not exist, unless $\alpha$ is a positive integer, because the integral in (24) is divergent for every $\theta \in[0,2 \pi[$. This has an important consequence: we cannot compute the derivative of a given function by using its Taylor series and computing the derivative term-by-term.

Let us see what happens for other values of $\beta$ and for $z$ in half-plane regions. The branch cut line needed for the definition of the function must be chosen to be outside the integration region. This is equivalent to say that the two branch cut lines cannot intersect. To use (24) we compute the successive integer order derivatives of this function that are given by

$D^{n} z^{\beta}=(-1)^{n}(-\beta)_{n} z^{\beta-n}$.

Now, we have

$$
\begin{aligned}
& D^{\alpha} z^{\beta} \\
& =\frac{\mathrm{e}^{j(\pi-\theta) \alpha}}{\Gamma(-\alpha)} \\
& \times \int_{0}^{\infty} \frac{\left[\left(x \cdot c^{j \theta}+z\right)^{\beta}-\sum_{0}^{\left.N \frac{(-1)^{n}(-\beta)_{n} z^{\beta-n}}{n !} \mathrm{e}^{j n \theta} x^{n}\right]}\right.}{x^{\alpha+1}} \mathrm{~d} x .
\end{aligned}
$$

With a substitution $\tau=x \cdot \mathrm{e}^{j \theta} / z$, we obtain:

$$
\begin{aligned}
D^{\alpha} z^{\beta}= & \frac{\mathrm{e}^{j \pi \alpha}}{\Gamma(-\alpha)} z^{\beta-\alpha} \\
& \times \int_{0}^{\infty \mathrm{e}^{j \theta / z}} \frac{\left[(1+\tau)^{\beta}-\sum_{0}^{N \frac{(-1)^{n}(-\beta)_{n}}{n !} \tau^{n}}\right.}{\tau^{\alpha+1}} \mathrm{~d} \tau .
\end{aligned}
$$

To simplify the analysis, let us assume that $\theta=0$ and $z \in R^{+}$. We obtain:

$D^{\alpha} z^{\beta}=\frac{\mathrm{e}^{j \pi \alpha}}{\Gamma(-\alpha)} z^{\beta-\alpha} \int_{0}^{\infty} \frac{\left[(1+\tau)^{\beta}-\sum_{0}^{N(-1)^{n}(-\beta)_{n}} \tau^{n}\right.}{\tau^{\alpha+1}} \mathrm{~d} \tau$.

As shown in $[5,6]$, the integral in (36) is a generalised version of the Beta function $B(-\alpha, \alpha-\beta)$ valid for $\alpha \in R$ and $\beta<0$. We conclude then that

$D^{\alpha} z^{\beta}=\frac{\mathrm{e}^{j \pi \alpha} \Gamma(\alpha-\beta)}{\Gamma(-\beta)} z^{\beta-\alpha}$

valid for any $\alpha$ and $\beta<0$ and for $z \in R^{+}$. Using the procedure described in [14] for dealing with the gamma function, it is not hard to show that the above formula is valid for $z \in C^{+}$.

This means that we have to use a branch cut line in the left half-plane. If $z \in C^{-}$, we choose $\theta=\pi$ and obtain the above result again. As one can be considered as an analytical continuation of the other, we can conclude that (37) is valid for $\beta<0$ and any $\alpha$, but we should be careful with the choice of the branch cut line. For $\beta>0$, the integral in (36) is divergent explaining the affirmation we did at the beginning of this section.

\subsection{The derivatives of real functions}

As we are mainly interested in real variable functions we are going to obtain the formulae suitable for this case. Now, we only have two hypotheses: $\theta=0$ or $\pi$. 


\subsection{1. $\theta=0$-reverse derivative}

This corresponds to choosing the real positive half-axis as branch cut line. Substituting $\theta=0$ into (24), we have:

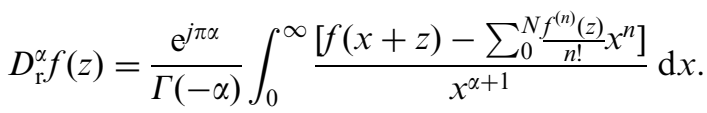

As this integral uses the right-hand values of the function, we will call this backward or reverse derivative in agreement with [9].

\subsection{2. $\theta=\pi$-direct derivative}

This corresponds to choosing the real negative half-axis as branch cut line. Substituting $\theta=\pi$ into (24) and performing the change $x \rightarrow-x$, we have:

$D_{\mathrm{d}}^{\alpha} f(z)=\frac{1}{\Gamma(-\alpha)} \int_{0}^{\infty} \frac{\left[f(z-x)-\sum_{0}^{N f^{(n)}(z)}(-x)^{n}\right]}{x^{\alpha+1}} \mathrm{~d} x$.

As this integral uses the left-hand values of the function, we will call this forward or direct derivative again in agreement with Ortigueira et al. [9].

\section{Derivatives of functions with Laplace and Fourier transforms}

Consider now the special class of functions with Laplace transform (LT). Let $f(t)$ be such a function and $F(s)$ its $\mathrm{LT}^{7}$, with a suitable region of convergence, $R_{c}$. This means that we can write

$f(t)=\frac{1}{2 \pi j} \int_{a-j \infty}^{a+j \infty} F(s) \mathbf{e}^{s t} \mathrm{~d} s$,

where $a$ is a real number inside the region of convergence. Inserting (40) inside (38) and permuting the integration symbols, we obtain:

$$
\begin{aligned}
D_{\mathrm{r}}^{\alpha} f(z)= & \frac{\mathrm{e}^{j \pi \alpha}}{2 \pi j \Gamma(-\alpha)} \int_{a-j \infty}^{a+j \infty} F(s) \mathrm{e}^{s z} \\
& \times \int_{0}^{\infty} \frac{\left[\mathrm{e}^{s x}-\sum_{0}^{N} \frac{(s x)^{n}}{n !}\right]}{x^{\alpha+1}} \mathrm{~d} x \mathrm{~d} s .
\end{aligned}
$$

If $\operatorname{Re}(s)<0$ and considering the result obtained in Section 4.1 (see $[14,18]$ also) the inner integral is equal to $\Gamma(-\alpha) \cdot s^{\alpha}$; if $\operatorname{Re}(s)>0$ it is divergent. We conclude that:

$$
\mathrm{LT}\left[D_{\mathrm{r}}^{\alpha} f(t)\right]=s^{\alpha} F(s) \quad \text { for } \operatorname{Re}(s)<0 .
$$

\footnotetext{
${ }^{7} \mathrm{We}$ assume the two-sided Laplace transform.
}

This means that there must exist an operator with transfer function equal to

$F(s)=s^{\alpha} \quad$ for $\operatorname{Re}(s)<0$.

This is an anti-causal operator with an impulse response equal to [7]

$\delta_{\mathrm{r}}^{(\alpha)}(t)=-\frac{t^{-\alpha-1} u(-t)}{\Gamma(-\alpha)}$,

where $u(t)$ is the Heaviside unit step. For the direct case, we use (39) and (40) to obtain again:

$$
\begin{aligned}
D_{\mathrm{d}}^{\alpha} f(z)= & \frac{1}{2 \pi j \Gamma(-\alpha)} \int_{a-j \infty}^{a+j \infty} F(s) \mathrm{e}^{s z} \\
& \times \int_{0}^{\infty} \frac{\left[\mathrm{e}^{-s x}-\sum_{0}^{N} \frac{(s x)^{n}}{n !}\right]}{x^{\alpha+1}} \mathrm{~d} x \mathrm{~d} s .
\end{aligned}
$$

If $\operatorname{Re}(s)>0$, the inner integral is equal to $\Gamma(-\alpha) . s^{\alpha}$; if $\operatorname{Re}(s)<0$ it is divergent. We conclude that:

$\mathrm{LT}\left[D_{\mathrm{d}}^{\alpha} f(t)\right]=s^{\alpha} F(s)$ for $\operatorname{Re}(s)>0$.

Again there must exist an operator with transfer function equal to

$F(s)=s^{\alpha} \quad$ for $\operatorname{Re}(s)>0$

and so, it is a causal operator with an impulse response equal to

$\delta_{\mathrm{d}}^{(\alpha)}(t)=\frac{t^{-\alpha-1} u(t)}{\Gamma(-\alpha)}$.

In Appendix we present the procedure for computing the inverse LT of $s^{\alpha}$. With this formulation we can conclude that we have a causal (forward) derivative that is the convolution of $f(t)$ and $\delta_{\mathrm{d}}^{(\alpha)}(t)$ :

$D_{\mathrm{d}}^{\alpha} f(t)=\frac{1}{\Gamma(-\alpha)} \int_{0}^{\infty} f(t-\tau) \cdot \tau^{-\alpha-1} \mathrm{~d} \tau$

valid for functions with LT converging in a region that includes the right-hand side of the complex plane. Similarly, we have an anti-causal (backward) derivative valid for functions with LT converging in a region that includes the left-hand side of the complex plane and that is the convolution of $f(t)$ and $\delta_{\mathrm{r}}^{(\alpha)}(t)$ :

$D_{\mathrm{r}}^{\alpha} f(t)=\frac{(-1)^{-\alpha}}{\Gamma(-\alpha)} \int_{0}^{\infty} f(t+\tau) \cdot \tau^{-\alpha-1} \mathrm{~d} \tau$.

It is interesting to remark that these definitions were introduced both exactly with this format by Liouville [10]. Unhappily in the common literature the factor $(-1)^{-\alpha}$ has been removed. To give the correct 
acknowledgement we will call (49) and (50) as Liouville forward and backward derivatives. ${ }^{8}$

To study the case of functions with Fourier transform, we can consider the results obtained in Section 4.1. However, we will use the derivatives just obtained. We begin by noting that the multivalued expression $F(s)=s^{\alpha}$ becomes an analytic function as soon as we fix a branch cut line in all the complex plane excepting the branch cut line. The computation of the derivative of functions with Fourier transform is dependent on the way used to define $(j \omega)^{\alpha}$. If we define it doing the limit as $s \rightarrow j \omega$ from the right which means that

$(j \omega)^{\alpha}=|\omega|^{\alpha} \begin{cases}\mathrm{e}^{j \alpha \pi / 2} & \text { if } \omega>0, \\ \mathrm{e}^{-j \alpha \pi / 2} & \text { if } \omega<0,\end{cases}$

we can use (46) and (49). If we perform the limit as $s \rightarrow j \omega$ from the left meaning that

$(j \omega)^{\alpha}=|\omega|^{\alpha} \begin{cases}\mathrm{e}^{j \alpha \pi / 2} & \text { if } \omega>0, \\ \mathrm{e}^{-j 3 \alpha \pi / 2} & \text { if } \omega<0,\end{cases}$

we are in the anti-causal case and use (43) and (50).

The derivative expressed in (49) is equal to (5), we can somehow easily obtain a strange relation:

$\frac{t^{-\alpha-1} u(t)}{\Gamma(-\alpha)}=\lim _{h \rightarrow 0+} \frac{\sum_{k=0}^{\infty}(-1)^{k}\left(\begin{array}{l}\alpha \\ k\end{array}\right) \delta(t-k h)}{h^{\alpha}}$.

For the reverse case, we obtain a similar result.

\section{Fractional linear systems}

The results of the previous section are very important in applications since they allow us to introduce the useful concept of transfer function. In fact, if we define a linear system through a fractional differential equation with the general format:

$\sum_{n=0}^{N} a_{n} D^{v_{n}} y(t)=\sum_{m=0}^{M} b_{m} D^{v_{m}} x(t)$,

where the differentiation orders, $v_{n}$, are, in the general case, complex numbers. As usual, we apply the LT to Eq. (54) and use the results of Section 5, to obtain the transfer function of the system:

$H(s)=\frac{\sum_{m=0}^{M} b_{m} s^{v_{m}}}{\sum_{n=0}^{N} a_{n} s^{v_{n}}}$

\footnotetext{
${ }^{8}$ The terms forward and backward are used here in agreement to the way the time flows, from past to future or the reverse.
}

with region of convergence defined by $\operatorname{Re}(s)>0$ (causal case) or $\operatorname{Re}(s)<0$ (anti-causal case). When looking for the output, $y(t)$, to a given input, $x(t)$, we must consider the initial conditions. This is a problem that created much confusion and difficulties in the past $[3,8,16]$ motivated by the use of several different derivative definitions and of the one-sided LT. In [8], we proposed a new way of looking at the problem. The proposed solution is based on the initial value theorem and on the Watson-Doetsch lemma:

The initial-value theorem Assume that $\varphi(t)$ is a causal signal such that in some neighbourhood of the origin is a regular distribution corresponding to an integrable function. Also, assume that there is a real number $\beta>-1$ such that $\lim _{t \rightarrow 0+} \varphi(t) / t^{\beta}$ exists and is a finite complex value. Then

$\lim _{t \rightarrow 0+} \frac{\varphi(t)}{t^{\beta}}=\lim _{\sigma \rightarrow \infty} \frac{\sigma^{\beta+1} \Phi(\sigma)}{\Gamma(\beta+1)}$.

For proof see [17].

Let us consider the class of functions with LT analytic for $R e(s)>\gamma$ that verify

$\varphi(t) \approx t^{\beta} \cdot \sum_{n=0}^{\infty} a_{n} \frac{t^{n v} u(t)}{\Gamma(\beta+1+n v)}$,

as $t \rightarrow 0+$ where $\beta>-1$ and $v>0$, where the principal values of the powers are assumed $v$ is greater than the maximum derivative order. The Watson-Doetsch lemma [14], states that the LT $\Phi(s)$ of $\varphi(t)$ satisfies:

$\Phi(s) \approx \frac{1}{s^{\beta+1}} \sum_{n=0}^{\infty} a_{n} \frac{1}{S^{n v}}$

as $s \rightarrow \infty$ and $\operatorname{Re}(s)>0$.

We will assume that the input and the output of the system described by (54) have the general format:

$f(t)=\sum_{n=0}^{L} f_{n}(t) t^{\gamma_{n}} u(t)$

where $0<\gamma_{n}<\gamma_{n+1} . L$ is a positive integer that may be infinite, and the functions $f_{n}(t)(n=0, \ldots, N)$ and their derivatives of orders less than or equal to $\gamma_{N}$ are assumed to be regular at $t=0$. We may assume them to be given by

$f_{n}(t)=\sum_{k=0}^{\infty} a_{k} \frac{t^{k v} u(t)}{\Gamma(\beta+1+k v)}$. 
Let us introduce a sequence $\beta_{n}$ by

$\beta_{n}=\gamma_{n}-\sum_{k=0}^{n-1} \beta_{k}, \quad \beta_{0}=\gamma_{0}$.

The solution of the initial conditions problem is obtained through the substitution of each derivative in (54) by

$\varphi^{\left(\gamma_{n}\right)}(t)=[f(t) \cdot u(t)]^{\left(\gamma_{n}\right)}-\sum_{i=0}^{n-1} f\left(\gamma_{i}\right)(0+) \delta_{\mathrm{d}}^{\left(\gamma_{n}-\gamma_{i}-1\right)}(t)$,

that states the general formulation of the initial value problem solution. As we can see, the initial values prolong their action for every $t>0$. This means that we have a memory about the initial conditions that decreases very slowly. Using the LT, we obtain:

$\operatorname{LT}\left[\varphi^{\left(\gamma_{n}\right)}(t)\right]=s^{\left(\gamma_{n}\right)} F(s)-s^{\gamma_{n}} \sum_{i=0}^{n-1} f^{\left(\gamma_{i}\right)}(0+) s^{-\gamma_{i}-1}$,

that is a generalisation of the usual formula for introducing the initial condition. The sequence $\gamma_{i}(i=0,1, \ldots, n)$ is chosen accordingly to the following rules:

(a) The $v_{n}$ on the left (or right) side in (54) belong to the sequence.

(b) The others are arbitrary unless the problem at hand suggests them.

(c) This means that we are almost free in choosing the intermediate derivatives between $\gamma_{n}$ and $\gamma_{n+1}$.

\section{Conclusions}

In this paper we proposed a new look at the fractional derivative by taking as starting point the Cauchy derivative formulation. We showed that it is equal to the Grünwald-Letnikov and performed its analysis by using the Hankel contour. From this analysis we obtained a regularised formulation that we used to obtain special formulae as the forward and backward derivatives. These allowed us to obtain the original Liouville formulae valid for functions with LT. It is interesting to remark that the most common definitions used nowadays for fractional derivatives, e.g. Riemann-Liouville and Caputo do not enter into the developed scheme.

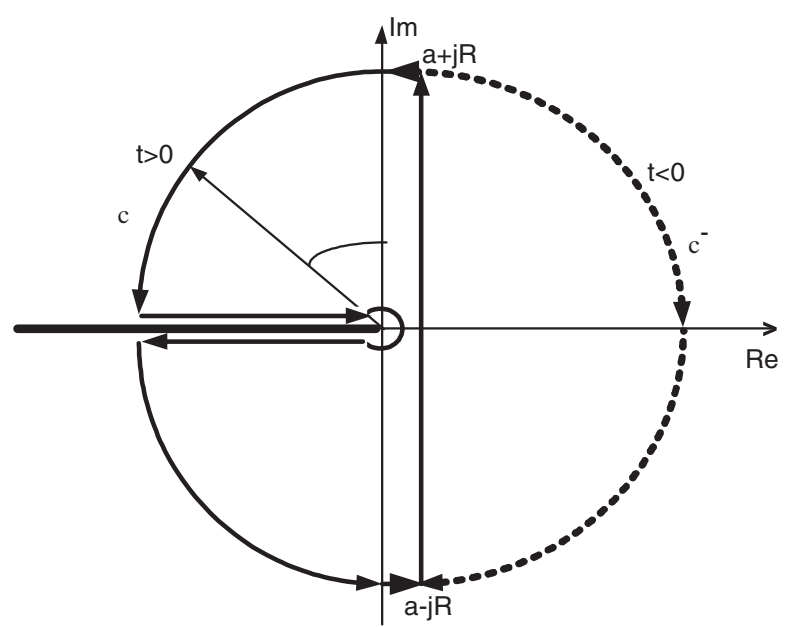

Fig. 3. Contours for Laplace transform inversion.

With this formulation we were able to introduce the fractional linear systems and the way out of introducing the initial conditions.

\section{Appendix. Inverting $\boldsymbol{s}^{\alpha}$}

We are going to compute the Inverse LT of $s^{\alpha}$, for $\alpha \in R$. We assume the causal case with region of convergence defined by $\operatorname{Re}(s)>0$. Let $\delta_{\mathrm{d}}^{\alpha}(t)$ be the causal Laplace inverse transform of $s^{\alpha}$. By simplicity, we can choose the negative real half-axis as branch cut and constrain the argument of $s$ to belong to the interval $]-\pi, \pi]$. Consider the picture in Fig. 3.

For $t<0$ we use the contour consisting of the segment of straight line and the closing dotted halfcircle. It is not hard to conclude that $\delta_{\mathrm{d}}^{\alpha}(t)=0$. For $t>0$ we use the bold integration path in Fig. 3. This has 8 segments: the inversion straight line, two short parallel straight line segments, two quarter circles, two parallel long straight line segments and a small circle. The integral computed along the total contour, $C$, is null since in its interior the function is analytic. It is not difficult to show that the integrals along the circle arcs and along the short segments tend to zero as $R \rightarrow \infty$. For the integrals over the long segments it is not difficult to conclude that their sum is equal to $2 j \sin \alpha \pi \cdot \int_{\rho}^{+\infty} x^{\alpha} \mathrm{e}^{-x t} \mathrm{~d} x$, $\rho$ being the radius of the small circle. To compute the integral over the small circle we proceed as in Section 2.1. From the Taylor series for the exponential, we conclude that it equals 
$-2 j \sin (\alpha \pi) \cdot \sum_{0}^{\infty}\left((-1)^{n} t^{n} \rho^{n+\alpha+1)}\right) /(n !(n+\alpha+1))$. If $\alpha$ is positive, the contribution of the small circle is zero as $\rho$ goes to zero. If $\alpha<0$, let $N$ be the least positive integer greater than $|\alpha|$. In this case, the contribution is merely equal to

$$
\begin{aligned}
- & 2 j \sin (\alpha \pi) \cdot \sum_{0}^{N} \frac{(-1)^{n} t^{n} \rho^{n+\alpha+1}}{n !(n+\alpha+1)} \\
& =-2 j \sin (\alpha \pi) \cdot \sum_{0}^{N} \frac{(-1)^{n} t^{n}}{n !} \int_{\rho}^{+\infty} x^{n-\alpha} \mathrm{d} x
\end{aligned}
$$

Joining this expression to the above one and letting $\rho$ go to zero, we obtain:

$\delta_{\mathrm{d}}^{\alpha}(t)=\frac{2 j \sin \pi \alpha}{2 \pi j} \cdot \int_{0}^{+\infty} x^{\alpha}\left[\mathrm{e}^{-x t}-\frac{(-1)^{n}(t x)^{n}}{n !}\right] \mathrm{d} x$

The use of the definition and properties of the gamma function [18] leads immediately to (35). For $t=0$, the inversion integral is not convergent. However, its principal value is zero.

\section{References}

[1] L.M.C. Campos, On a concept of derivative of complex order with applications to special functions, IMA J. Appl. Math. 33 (1984) 109-133.

[2] L.M.C. Campos, Fractional calculus of analytic and branched functions, in: R.N. Kalia (Ed.), Recent Advances in Fractional Calculus, Global Publishing Company, 1993.

[3] K.S. Miller, B. Ross, An Introduction to the Fractional Calculus and Fractional Differential Equations, Wiley, New York, 1993.

[4] K. Nishimoto, Fractional Calculus, Descartes Press Co., Koriyama, 1989.
[5] M.D. Ortigueira, From differences to differintegrations, Fractional Calculus Appl. Anal., in preparation.

[6] M.D. Ortigueira, Two new integral formulae for the Beta function, Int. J. Appl. Math., in preparation.

[7] M.D. Ortigueira, Introduction to Fractional Signal Processing. Part 1: Continuous-Time Systems, IEE Proc. Vision Image Signal Process. 1 (2000) 62-70.

[8] M.D. Ortigueira, On the initial conditions in continuoustime fractional linear systems, Signal Process. 83 (11) (November 2003) 2301-2309.

[9] M.D. Ortigueira, J.A. Tenreiro-Machado, J. e Sá da Costa, Which differintegrator?, IEE Proc. Vision Image Signal Process. 152 (6) (2005) 846-850.

[10] S. Dugowson, Les différentielles métaphysiques, Ph.D. Thesis, Université Paris Nord, 1994.

[11] J.B. Diaz, T.J. Osler, Differences of Fractional Order, Math. Comput. 28 (125) (January 1974).

[12] M.D. Ortigueira, Fractional differences integral representation and its use to define fractional differintegrations, in: Proceedings of the ENOC-2005, Fifth EUROMECH Nonlinear Dynamics Conference, Eindhoven University of Technology, The Netherlands, 7-12 August 2005.

[13] M.D. Ortigueira, A new look at the differintegration definition, in: Proceedings of the ENOC-2005, Fifth EUROMECH Nonlinear Dynamics Conference, Eindhoven University of Technology, The Netherlands, 7-12 August 2005.

[14] P. Henrici, in: Applied and Computational Complex Analysis, vol. 2, Wiley, New York, 1991, pp. 389-391.

[15] S.G. Samko, A.A. Kilbas, O.I. Marichev, Fractional Integrals and Derivatives - Theory and Applications, Gordon and Breach Science Publishers, London, 1987.

[16] I. Podlubny, Fractional Differential Equations, Academic Press, San Diego, 1999.

[17] A.H. Zemanian, Distribution Theory and Transform Analysis, Dover Publications, New York, 1987.

[18] M.A. Chaudhry, S.M. Zubair, On a Class of Incomplete Gamma Functions with Applications, Chapman \& Hall, London, CRC, Boca Raton, FL, 2002. 